\title{
Gradhiva
}

\section{Alain Dierkens, Gil Bartholeyns et Thomas Golsenne (éd.), La Performance des images}

Bruxelles, Éditions de l'université de Bruxelles, coll. « Problèmes

d'histoire des religions »19, 2009, $258 \mathrm{p}$.

\section{Giordana Charuty}

\section{(2) OpenEdition}

Journals

Édition électronique

URL : http://journals.openedition.org/gradhiva/2095

DOI : 10.4000/gradhiva.2095

ISSN : 1760-849X

Éditeur

Musée du quai Branly Jacques Chirac

Édition imprimée

Date de publication : 18 mai 2011

Pagination : 226-227

ISBN : 978-2-35744-042-5

ISSN : 0764-8928

Référence électronique

Giordana Charuty, « Alain Dierkens, Gil Bartholeyns et Thomas Golsenne (éd.), La Performance des images », Gradhiva [En ligne], 13 | 2011, mis en ligne le 18 mai 2011, consulté le 21 septembre 2020 URL : http://journals.openedition.org/gradhiva/2095; DOI : https://doi.org/10.4000/gradhiva.2095

Ce document a été généré automatiquement le 21 septembre 2020.

(C) musée du quai Branly 


\section{Alain Dierkens, Gil Bartholeyns et Thomas Golsenne (éd.), $L a$ Performance des images}

Bruxelles, Éditions de l'université de Bruxelles, coll. « Problèmes d'histoire des religions »19, 2009, 258 p.

\section{Giordana Charuty}

\section{RÉFÉRENCE}

Alain Dierkens, Gil Bartholeyns et Thomas Golsenne (éd.), La Performance des images.

Bruxelles, Éditions de l'université de Bruxelles, coll. « Problèmes d'histoire des religions $» 19,2009,258 \mathrm{p}$.

1 Résultat de rencontres entre médiévistes de l'Université libre de Bruxelles et de l'École des hautes études en sciences sociales de Paris, ce volume de la collection « Problèmes d'histoire des religions », dirigéepar Alain Dierkens, succède aux nombreux colloques et publications consacrés, depuis les années 1980, au rôle des images dans le monde médiéval. Comme le rappelle Jérôme Baschet dans un bref prologue, les études de cas se sont multipliées pour dresser l'inventaire de ce que « fait » et « fait faire » l'image dans la culture médiévale (p.12), en même temps que, dans une perspective d'anthropologie historique, les chercheurs se sont employés à renouveler leurs modèles conceptuels pour décrire les modalités d'animation et d'efficacité de ces objets.

2 Ainsi, la réflexion épistémologique qui accompagne les études rassemblées par Gil Bartholeyns et Thomas Golsenne a pour ambition d'introduire des distinctions analytiques dans la description de ces "actes d'images" que le succès du terme " pouvoir ", consacré par la réception internationale du livre de David Freedberg - The Power of Images. Studies in the History and Theory of Response (1989) - pouvait conduire à traiter de manière uniforme. Pour saisir cette performance iconique dans sa spécificité médiévale, il convient, selon les auteurs, de distinguer quatre notions - agentivité, 
efficacité, performativité, puissance - sous lesquelles sont distribuées les contributions, selon qu'elles privilégient l'une ou l'autre de ces questions: «1) En quelle manière les images sont-elles des êtres vivants ? 2) Quels sont les effets des images ? 3) Quelles sont les modalités des actes d'images? 4) Que peut une image?»(p. 24-25). Cependant, toutes prennent à la fois en compte l'élaboration normative par la pensée théologique et juridique, et une diversité d'usages - dispositifs cultuels, usages vestimentaires, ornementations peintes, sculptées, enluminées - où, comme le rappelle Gil Bartholeyns, la théorie médiévale du signe et la théorie chrétienne des images, pour aussi subtiles soient-elles, «font partie du "problème", plus que de la solution » (p. 138). Jean-Claude Schmitt rappelle, en ouverture d'une première partie centrée sur l'animation des images, qu'à partir de l'an mil celles-ci, comme les lieux, les temps, les personnes, font l'objet de procédures codifiées de consécration qui hiérarchisent les degrés de sacralité qu'elles auront à parcourir. Les images deviennent miraculeuses question reprise par Jean-Marie Sansterre à partir d'études antérieures sur des récits des $\mathrm{X}^{\mathrm{e}}$-XIII ${ }^{\mathrm{e}}$ siècles - lorsqu'elles sont investies et activées par le prototype divin qui vient «habiter temporairement sa représentation» (p.57). Tout autant que l'être figuré, c'est la parure qui, pour Thomas Golsenne, fait l'image cultuelle en convertissant l'efficacité tactile en efficacité visuelle (p. 82). Quant aux nombreuses images animales, on ne saurait généraliser l'intentionnalité symbolique des bestiaires. Reprenant la proposition de Meyer Shapiro, Pierre-Olivier Dittmar suggère de lire celles sculptées à l'extérieur des églises en termes, non de sens, mais de force, leur effet ornemental visant à en renforcer la protection (p.68).

4 Sous la rubrique "Efficacité », Brigitte d'Hainaut-Zveny décrit la promotion des images religieuses en théâtre didactique, substitut efficace des reliques, qui marque le passage des retables romans aux retables gothiques avant de laisser place, dans les autels renaissants, à la réserve eucharistique qui fait jouer à la matière sacramentelle la fonction de relique unique, sous l'influence de la Contre-Réforme. Mais adopter le vocabulaire de la performance suffit-il à renouveler, dans une perspective anthropologique, une iconographie somme toute très classique? Plus convaincante sous ce rapport, apparaît la belle étude qu'Elisa Brilli consacre à un tableau - l'Allegoria della Commedia - commandé en 1465 à Domenicho Di Michelino, comme substitut du monument funéraire à la gloire de Dante (exilé et mort à Ravenne en refusant toute amnistie) que la commune de Florence ne s'est pas résolue à construire. Ce « mémorial peint ", selon l'expression empruntée à Georges Didi-Huberman, qui orne toujours le bas-côté nord du dôme, actualise le retour à Florence et le couronnement du poète par une cité idéale en jouant, montre-t-elle, de la double efficacité du discours figuratif (celle du médium et celle de son contenu sémantique) pour faire prévaloir l'auctoritas de la ville sur celledu poète lui-même.

Parler de performativité de l'image appelle, bien sûr, la comparaison avec les actes linguistiques, en particulier avec la première formulation qu'en a donnée Austin : est performatif un énoncé qui fait advenir la réalité qu'il représente. Encore doit-on prendre en compte l'imposante réflexion médiévale sur les diverses catégories de signes. La reformulation par Thomas d'Aquin du bien-fondé du culte des images sur des bases aristotéliciennes invite Jean Wirth à admettre la notion d'" acte iconique " par analogie avec l'acte de parole car, sans être un performatif complet, l'image-signe, par opposition à l'image-objet, donnerait au processus performatif «une force particulière ». Pourtant, à suivre l'analyse de ces signes sociaux que sont les vêtements, 
une tension constante traverse la culture médiévale, laquelle se traduit par une succession de renversements sur le plan des discours, sinon dans l'ordre de la pratique (Gil Bartholeyns). L'Église, entre les $\mathrm{V}^{\mathrm{e}}$ et $\mathrm{IX}^{\mathrm{e}}$ siècles, ne cesse de rappeler que les actes valent mieux que les symboles d'identification. Mais la codification formelle et l'imposition du vêtement ascétique vont rapidement être justifiées en termes "performatifs" - vivons selon notre habit, ordonnent au milieu du $\mathrm{VI}^{\mathrm{e}}$ siècle les Instructions de Dorothée de Gaza - ouvrant, ainsi, la voie à l'institution de la «prise d'habit » comme sacrement. Cependant, dans une société saturée d'emblèmes et de symboles, pensant comme conventionnelles aussi bien la signification que l'efficacité des signes, une grande part de la réflexion du XIII e siècle, d'inspiration augustinienne, sur le faux et le mensonge des apparences sociales conduit à refuser sur le plan éthique cette performativité des signes de l'identification sociale (p. 149).

6 Aux rapports complexes de l'image et de l'écriture sont consacrées plusieurs des contributions rassemblées sous les rubriques de la performativité et de la puissance. Les pratiques contemporaines du montage cinématographique et des performances multimédia sont convoquées pour décrire les effets de lecture produits par les manuscrits enluminés de théâtre médiéval ou de la Légende dorée (Corneliu Dragomirescu, Chloé Maillet). Mais l'étude la plus passionnante est, sans nul doute, celle, pourtant très technique, que Jean-Claude Bonne et Eduardo Aubert consacrent aux relations entre l'image et l'annotation du chant dans un manuscrit de la fin du $\mathrm{x}^{\mathrm{e}}$ siècle. L'hypothèse séduisante avancée par l'historien et le musicologue est que les étonnantes images d'instrumentistes, de danseurs et de jongleurs montrent, non la voix elle-même, mais « le genre de performances orales " prescrit par l'annotation musicale. Ici, la notion de performativité est pleinement justifiée dans la mesure où elle n'est pas introduite, a posteriori, dans des analyses d'abord conduites à l'aide d'autres modèles conceptuels, mais qu'elle commande la description rigoureuse des choix figuratifs et scripturaires, dans leurs plus infimes détails, et permet de comprendre l'engagement du lecteur chanteur médiéval et d'apprendre, au lecteur moderne, à lire cette transposition visuelle d'une modalité d'exécution vocale.

7 La distribution des études entre les diverses modalités de l'agir iconique paraît, somme toute, relativement arbitraire, car l'ensemble du questionnement se trouve inévitablement posé, plus ou moins explicitement, à tous les cas examinés, lesquels débordent la culture médiévale en incluant quelques usages photographiques (André Gunthert, Pierre Lagrange). Et, comme le souligne en épilogue Irène Rosier-Catach, les auteurs considèrent de manière presque exclusive le rapport de l'image et du récepteur, au détriment de l'émetteur, faisant ainsi des réactions produites par l'image l'accès privilégié pour en décréter l'efficience (p. 251). Ce parti pris était déjà celui de David Freedberg, sans doute trop rapidement rangé par Gil Bartholeyns et Thomas Golsenne parmi les représentants d'une neuro-esthétique travaillant à une " naturalisation du rapport aux images " (p.18), à laquelle s'opposerait le parti pris « culturaliste » des historiens médiévistes réunis ici. Le colloque international qui vient de se tenir à Noto (Sicile) - "Formes et modèles. La photographie comme mode de connaissance » (7-9 octobre 2010) - à l'initiative de la Société italienne pour l'étude de la photographie et du département de sciences cognitives et d'études culturelles de l'université de Messine, a, justement, permis à David Freedberg de répondre aux objections des anthropologues, en explicitant et nuançant certaines de ses affirmations 
antérieures concernant les rapports nécessaires entre histoire des images et sciences cognitives, question à laquelle il entend consacrer un nouvel ouvrage. À suivre, donc.

\section{AUTEURS}

\section{GIORDANA CHARUTY}

giordana.charuty@laposte.net 